\title{
Phonons in an aperiodic alkane/urea composite crystal studied by inelastic x-ray scattering
}

\author{
C. Ecolivet,,${ }^{1, *}$ M. Verezhak,${ }^{1,2, \dagger}$ C. Mariette,${ }^{1}$ L. Guérin, ${ }^{1}$ P. Rabiller, ${ }^{1}$ J. Ollivier, ${ }^{3}$ A. Bosak,${ }^{4}$ and B. Toudic ${ }^{1}$ \\ ${ }^{1}$ Univ Rennes, CNRS, IPR (Institut de Physique de Rennes) - UMR 6251, F-35000 Rennes, France \\ ${ }^{2}$ Paul Scherrer Institute, $\mathrm{CH}-5232$ Villigen, Switzerland \\ ${ }^{3}$ Institut Laue-Langevin, 6 rue Jules Horowitz, F-38042 Grenoble, France \\ ${ }^{4}$ European Synchrotron Radiation Facility, BP 220, F-38043 Grenoble Cedex, France
}

(Received 23 August 2018; revised manuscript received 31 October 2018; published 21 December 2018)

\begin{abstract}
Aperiodic composite crystals belong to a new state of matter that possesses long-range order without translational symmetry. We report measurements of low-lying phonon branches in an $n$-alkane/urea compound that provide insight into the urea sublattice and the commensurate basal plane dynamics. Focusing on the thoroughly studied $n$-nonadecane/urea compound makes a comparison with other inelastic scattering results possible, allowing the observation of two distinct aperiodic elastic signatures. Using three techniques, a slight but reproducible difference in sound velocities is observed between the transverse mode propagating along the aperiodic direction and polarized in the commensurate basal plane, and the one polarized along the aperiodic direction propagating in the basal plane.
\end{abstract}

DOI: 10.1103/PhysRevB.98.224308

\section{INTRODUCTION}

Phonon spectroscopy is widely used to obtain detailed information on the interatomic or intermolecular interactions in condensed matter, from the simplest regular crystals to more complex structures. Contrary to light scattering, neutron and x-ray inelastic scattering permits investigations of excitations with very large wave vectors. Having a lower resolution than with cold neutrons, $x$-ray scattering nevertheless allows studies with a constant resolution even far away from the null wave vector. It is then easier to get a global point of view of the lattice dynamics of a given structure.

Aperiodic crystals are long-range ordered materials that recover their periodicity in a higher-dimensional space [1]. The diffraction patterns of these aperiodic materials exhibit sharp Bragg peaks characteristic of long-range order, although the systems are not invariant under spatial translations of the lattice [2]. The dimension (or rank) of the higher-dimensional space corresponds to the number of independent vectors required to describe the entire set of diffraction Bragg peaks (usually four, five, or six). The crystallographic superspace that describes such materials thus decomposes into two orthogonal subspaces: the usual physical space and an internal one. These materials are conventionally divided into three families: quasicrystals, incommensurately modulated crystals, and composite intergrowth crystals. Incommensurately modulated crystals are the simplest of the three since they always stem from a prototype periodic high-symmetry (HS) phase. Furthermore, the incommensurate phase can generally be treated as a perturbation of this periodic HS phase [3]. As an important consequence, a mean Brillouin zone is defined as being the HS zone and collective excitations can be readily

\footnotetext{
*ecolivet@univ-rennes1.fr

†mariana.verezhak@psi.ch
}

calculated as for $A_{2} B X_{4}$ compounds [4]. Away from the irrational critical wave vector $\mathbf{q}_{\mathbf{s}}$, which characterizes any incommensurate phase, the phonons are considered similarly to the HS phase. Around $\mathbf{q}_{\mathrm{s}}$ a perturbation calculation shows that phonons separated by $2 \mathbf{q}_{\mathrm{s}}$ are coupled by static incommensurate modulation. The nature of two phonons around $+\mathbf{q}_{\mathrm{s}}$ and $-\mathbf{q}_{\mathbf{s}}$ changes into two distinct excitations, called the phason and amplitudon, respectively, corresponding to phase and amplitude vibrations of the modulation. The phason modes are represented by collective excitations along the internal (supplementary) dimensions of the superspace. In the assumption of continuous incommensurate modulation functions, their branches present an acousticlike linear dispersion from $+\mathbf{q}_{\mathbf{s}}$ and $-\mathbf{q}_{\mathbf{s}}$. However, these modes are not true Goldstone modes since they are dissipative and thus appear as overdamped modes at $+\mathbf{q}_{\mathrm{s}}$ and $-\mathbf{q}_{\mathbf{s}}$.

Such excitations have been directly observed by neutron scattering in a few compounds, some compiled first in reviews $[5,6]$ and later in another organic compound [7]. These excitations are now much better understood, either from the point of view of Landau structural phase transitions or by using microscopic models $[1,3,8]$. In contrast, incommensurate composite crystals are composed of at least two interpenetrating modulated crystalline subsystems that are incommensurate in at least one direction and neither a mean structure nor a mean Brillouin zone may be defined in the physical space at three dimensions [9]. Consequently, no simple perturbation theory of the collective dynamics can be developed. This is one of the reasons for a continuing interest in aperiodic intergrowth compounds [10]. Some of them are expected to present unusual physical properties such as lubrication between out of register surfaces, as indicated by several theoretical studies [11-13]. However, observations of the expected behaviors in reality are rather scarce, which is probably due to nonideal realizations.

Among such compounds, those possessing a structure with a one-dimensional aperiodicity are conceptually simpler and 
allow, in diffraction experiments, an easy discrimination of Bragg peaks of the different sublattices along the aperiodic direction, along which different dynamics in composite structures are expected when the sublattices differ elastically. The intrinsic vibrational responses of each sublattice can be observed at large wave vectors. Previous studies of phonons at nonzero wave vectors were reported in a few incommensurate composites. Actually, they revealed acoustic phonons of different sublattices with a strong dependence on the nature of the related Bragg peak: mercury chains in $\mathrm{AsF}_{6}$ [14], selfhosting phases in $\mathrm{Rb}$ IV under pressure [15], superconducting cuprates such as $\mathrm{Bi}-2212$, and spin-ladder systems $[10,16]$ using inelastic neutron (INS) and/or inelastic x-ray scattering (IXS). At infinite wavelengths, these vibrational responses should transform, on one hand, into a regular acoustic mode, involving in-phase displacements of both sublattices, and, on the other hand, into a phase mode characterized by outof-phase displacements, similarly to an optic mode. In the case of weak intermodulations, the latter may be imagined by a relative sliding motion of the sublattices: the sliding mode, which is also the phase mode similar to the phason in modulated phases. The characteristics of these modes have been thoroughly analyzed $[13,17,18]$.

The prototype example for such studies is $\mathrm{HgAsF}_{6}$, where a crossover from the high-energy dispersion of the two longitudinal modes to a low-energy mixing was expected to give rise to the longitudinal sound mode and the sliding mode [19]. A complex feature was also reported in $n$-nonadecane/urea that was assigned to the coupling of the longitudinal acoustic phonon of the host urea sublattice and the predicted sliding mode [20]. However, direct unambiguous observations by either neutron, x-ray, or light scattering of underdamped sliding modes have yet to be reported. Sliding modes are not the unique signature of aperiodic composite structures since elastic properties are also modified by aperiodicity; for example, in acoustic modes polarized along aperiodicity directions, displacements of the different sublattices may be decoupled as shown in Ref. [18].

In the case of $n$-alkane/urea compounds, which typically belong to the host-guest family, the honeycomblike urea sublattice is very stiff due to hydrogen bonding. However, it is unstable versus a basal shear strain as proven by the alkane removal from the channels that leads to the formation of the pure urea tetragonal structure. At room temperature, the pure $n$ alkane guests may be liquid for the shortest molecules [21,22] or in rotatory phases characterized by strong conformational, translational, and rotational disorders similar to plastic crystal phases. When densely packed by cocrystallization inside the urea channels, an intermodulated aperiodic crystal is usually obtained, as illustrated in Fig. 1. In the restricted size of these channels, the alkane molecules are linear with a small amount of gauche conformers at their extremities [23] and, at room temperature, they present rotational and translational degrees of disorder. This family of crystals gives a prototypical example of symmetry breaking within crystallographic superspaces. For nanotubular structures with a single incommensurate direction $c$, a four-dimensional superspace description gives the positions of all of the Bragg peaks [24,25],

$$
\mathbf{G}_{h k l m}=h \mathbf{a}^{*}+k \mathbf{b}^{*}+l \mathbf{c}_{\mathbf{h}}^{*}+m \mathbf{c}_{\mathbf{g}}^{*},
$$
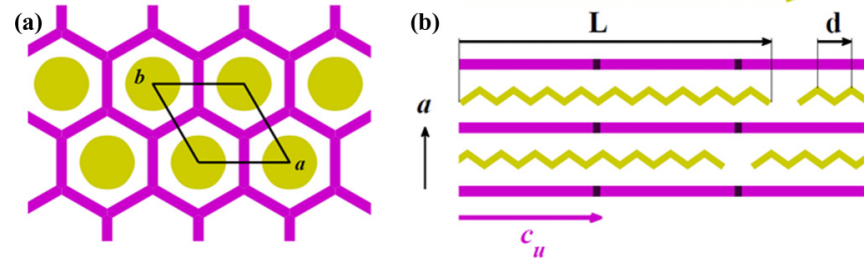

FIG. 1. Schematic representation of the host/guest crystal of $n$ nonadecane/urea at room temperature, with the hexagonal unit cell vectors $\mathbf{a}, \mathbf{b}, \mathbf{c}_{\text {host }}, \mathbf{c}_{\text {guest }}$ and the characteristic parameters of the $n$-nonadecane $\mathrm{CH}_{3}-\left(\mathrm{CH}_{2}\right)_{17}-\mathrm{CH}_{3}$ molecule (internal periodicity $d$ of the $\mathrm{C}_{2} \mathrm{H}_{4}$ and length $L$ defining respectively the position and the width of the intensity of the molecular form factor, the broad $D$ bands).

where $\mathbf{a}^{*}, \mathbf{b}^{*}, \mathbf{c}_{\mathbf{h}}^{*}$, and $\mathbf{c}_{\mathbf{g}}^{*}$ are the conventional reciprocal unit cell vectors, and $\mathbf{c}_{\mathbf{h}}^{*}$ and $\mathbf{c}_{\mathbf{g}}^{*}$ refer to the host and the guest parameters. Four indices $(h, k, l$, and $m)$ are needed to describe the four different types of structure Bragg peaks. A convenient but simplistic assignment is that the $h k 00$ peaks are common Bragg peaks, the $h k l 0$ and $h k 0 m$ correspond to the host and guest, respectively, whereas the $h \mathrm{klm}$, with $l$ and $m$ both different from zero, are intermodulation satellite Bragg peaks. However, due to sublattice intermodulation, the intensities of the host and guest Bragg peaks exhibit contributions from both substructures.

Among this series, the $n$-nonadecane/urea has been extensively studied in relation to its structural transitions versus temperature and pressure [26-28]. The room-temperature space group of $n$-nonadecane/urea is in dimension 4: $P 6_{1} 22(00 \gamma)$. The parameters of the hexagonal lattice are $a=$ $b=8.22 \AA, c_{\text {host }}=11.02 \AA$, and $\gamma$ is the misfit parameter defining $\mathbf{q}_{\mathbf{s}}=\gamma \cdot \mathbf{c}_{\mathbf{u}}^{*}$, with $\gamma=c_{\text {host }} / c_{\text {guest }}=c_{\mathrm{u}} / c_{\mathrm{a}}$. Gamma is found to be equal to 0.418 in the deuterated structure in contrast to 0.412 in the unlabeled compound. The existence of a $6_{1}$ helical axis in the high-symmetry phase strongly reduces the number of Bragg peaks present along the purely longitudinal $\left(00 c^{*}\right)$ reciprocal line, as seen in Fig. 1. The dynamics of this crystal has been investigated by nuclear magnetic resonance (NMR), light, and neutron scattering techniques, revealing unusual features [20,29,30]. Despite its one-dimensional simplicity, the possibility of obtaining a complete overview of the collective dynamics of this aperiodic material has not been permitted up to now. IXS, with its potential large scattering wave vector at a constant resolution, provides a unique opportunity to get a wider scope on the lattice dynamics of this compound.

\section{EXPERIMENT}

IXS measurements were performed on the ID28 spectrometer at the European Synchrotron Radiation Facility (ESRF). The optical layout is based on the triple-axis principle. The very high-energy resolution is obtained by a monochromator, operating at a Bragg angle very close to $90^{\circ}$, i.e., close to backscattering geometry. The scattered photons are energy analyzed by a perfect spherical silicon crystal analyzer, operated in Rowland geometry, and at a Bragg angle of $89.98^{\circ}$. ID28 

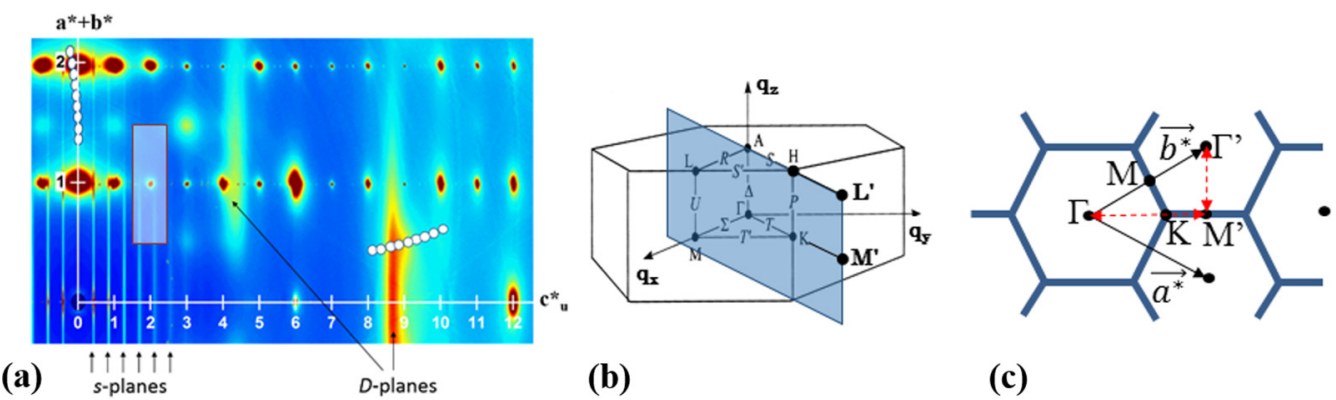

(c)

FIG. 2. (a) Diffraction image of the $\left(\mathbf{a}^{*}+\mathbf{b}^{*}, \mathbf{c}^{*}\right)$ reciprocal plane of $n$-nonadecane/urea as measured on ID29 at $T=300 \mathrm{~K}$, revealing a quarter of the accessible zone in the reciprocal space when using the spectrometer ID28 with the wavelength $\lambda=0.54 \AA$. The vertical axis scale gives the $h=k$ values whereas the $l$ values are on the horizontal scale in $\mathbf{c}_{\mathbf{u}}^{*}$ units. Along $\mathbf{c}^{*}$, only urea peaks with $6 \mathbf{c}_{\mathbf{u}}^{*}$ and $12 \mathbf{c}_{\mathbf{u}}^{*}$ are visible whereas common peaks at $\mathbf{a}^{*}+\mathbf{b}^{*}$ and $2\left(\mathbf{a}^{*}+\mathbf{b}^{*}\right)$ are accessible. Note the presence of diffuse scattering. The elongated rectangle is the crosscut of the first Brillouin zone of the urea sublattice by this scattering plane. The white dots are the location of the nine detectors for two different $Q$ vectors. (b) Hexagonal first urea Brillouin zone: The blue rectangle corresponds to the crosscut of the Brillouin zone in (a), with half a zone edge side of the neighboring Brillouin zones on each side. The irreducible first Brillouin zone is the prism $\Gamma K M L A H$, where $L^{\prime}$ and $M^{\prime}$ are equivalent to $L$ and $M$. (c) In order to present the (hk0) plane, an image of one first Brillouin zone and its neighbor in the ( $\left.h k 0\right)$ plane shows the main symmetry points, the $\mathbf{a}^{*}$ and $\mathbf{b}^{*}$ axes, and the directions shown in Fig. 5 (in red).

benefits from nine analyzers placed on a movable arm, $7 \mathrm{~m}$ in length. The $\mathrm{x}$ rays diffracted from the analyzer crystal are recorded by solid-state detectors (low-noise silicon detectors) that provide a very low background. As a compromise between the energy resolution and the beam flux, the (12 12 12) configuration was retained, yielding an incident photon wavelength of $\lambda=0.5226 \AA$ ( $E \simeq 23 \mathrm{keV})$ and allowing an energy resolution of $\sim 1.5 \mathrm{meV}$ [31] in a wide part of the reciprocal space up to $\sim 10 \AA^{-1}$. The organic nature of the sample $\mathrm{C}_{19} \mathrm{H}_{40} / \mathrm{CO}\left(\mathrm{NH}_{2}\right)_{2}$ requires a large single crystal, in this case $0.5 \times 0.5 \times 1 \mathrm{~cm}^{3}$, in order to maximize the scattered intensity.

Inelastic scattering is characterized by the transfer vector $\mathbf{Q}$ equal to the difference between the incident and scattered beam wave vectors. Both its magnitude and direction are important since the inelastic intensity scattered by a phonon with a polarization e varies as $(\mathbf{e} \cdot \mathbf{Q})^{2}$. Phonon wave vectors are defined by $\mathbf{Q}=\mathbf{G}_{h k l m}+\mathbf{q}$. Most of the experiments were performed within the scattering plane $\left(\mathbf{a}^{*}+\mathbf{b}^{*}, \mathbf{c}^{*}\right)$. This plane is shown in Fig. 2, as reconstructed from diffraction data obtained at room temperature on the beamline ID29 at the ESRF. At $\mathbf{G}_{h k l m}=0$, Bragg reflections are common to both sublattices, whereas the other points of the $\left(\mathbf{a}^{*}+\mathbf{b}^{*}, \mathbf{c}^{*}\right)$ plane may be distinguished with respect to the sublattice periodicity. All strong Bragg peaks may be assigned to the urea sublattice. This figure also shows that diffuse scattering occurs at many locations in this plane with three different origins outside the Bragg locations. Very narrow parallel streak lines perpendicular to the $c^{*}$ axis, called $s$ planes, define the reciprocal periodicity of $n$-nonadecane $\left(0.412 c_{u}^{*}\right)$. They are the fingerprint of a static lateral translational disorder in this sublattice. Also in relation to the finite length $L$ of the alkanes, but due to their internal periodicity $d$ of the $\mathrm{C}_{2} \mathrm{H}_{4}$ species, as defined in Fig. 1, broader lines ( $D$ planes) appear at $q_{z}=4.34 c_{u}^{*}$ and $8.68 c_{u}^{*}$ created by the dynamical translational and rotational disorder of the alkanes [32,33]. Finally, pretransitional diffuse scattering grows around locations $(h+$ $0.5 k+0.5 l \mathrm{~m})$, as in (1.5 1.53 0) located close to the upperright-hand corner of the gray rectangle on Fig. 2(a), where superstructure Bragg peaks appear at lower temperatures [34]. At small wave vectors, close to strong Bragg peaks, acoustic modes are expected to be strong scatterers, but since only a few faint Bragg peaks with an alkane periodicity appear, all attempts to record inelastic spectra in these locations during a reasonable time remained unsuccessful. Consequently, for $q_{z} \neq 0$ nearly all the observed inelastic peaks are related to the dynamics of the urea sublattice, which is a three-dimensional (3D) (modulated) long-range ordered structure connected by well-defined hydrogen bonds between urea molecules.

Even if both sublattices contribute to the intensity of any Bragg peak, the guest sublattice contribution, located at the urea reciprocal positions, is in proportion to its modulated fraction, which is not expected to be significant. So, in order to get a broad overview of the inelastic modes we will begin by the simplest assumption that the observed dynamics out of the basal plane is mainly due to urea, with only some weak perturbations. Consequently, we may use a Brillouin zone defined by the hexagonal urea reciprocal sublattice; its trace in the scattering plane appears as a rectangle elongated along the ( $\left.\mathbf{a}^{*}+\mathbf{b}^{*}\right)$ direction [Fig. 2(b)]. It should be noticed that the area beyond the $\pm K$ reciprocal points, i.e., $0.33<$ $\|q\|<0.5$, is in fact at the Brillouin zone boundary. The nine detectors may cover several Brillouin zones, by reducing all the detector locations inside the first irreducible Brillouin zone, we obtained ca. 240 nonequivalent phonon wave vectors scattered inside such a zone. The related inelastic spectra have been fitted by the convolution of damped harmonic oscillators (DHOs) and/or relaxation profiles weighted by the Bose factor with the apparatus function, which is a weighted sum of a Gaussian and a Lorentzian function with typical full width at half maxima (FWHM) of $\sim 1$ and $3 \mathrm{meV}$, respectively. The weight of the Lorentzian contribution is in the $10 \%$ range. These parameters vary somewhat with the analyzer used.

Spectra were usually recorded in a range of a few tens of $\mathrm{meV}$ in steps of $0.3 \mathrm{meV}$; each data point lasted $30 \mathrm{~s}$, sufficient to have manageable data statistics. Figure 3 displays typical spectra of different kinds of phonons propagating along the aperiodic channel direction $(\mathbf{q} \| \Gamma A)$ or in the basal 


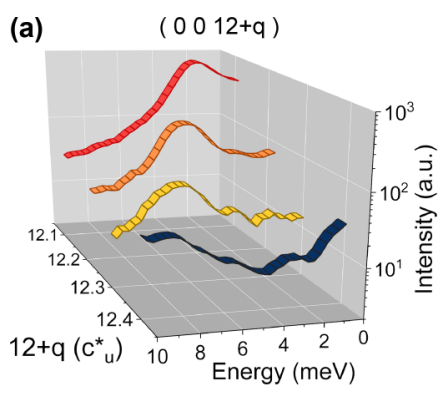

(b) ( q q 12 )

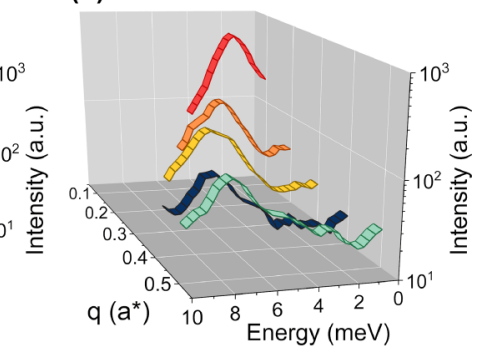

(c)

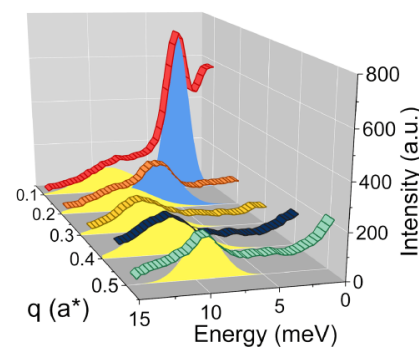

(d)

( 22 q)

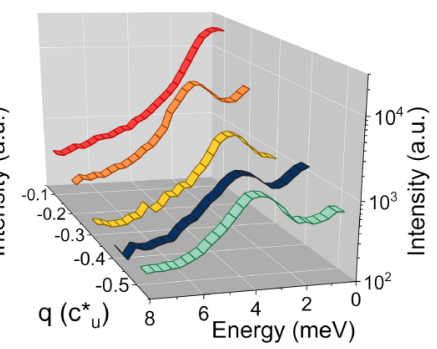

FIG. 3. Raw IXS spectra from phonons propagating along different symmetry directions. Titles indicate $\mathbf{Q}$ components in the reciprocal urea frame whereas $(q q)$ indicates relative equal components along the $\mathbf{a}^{*}, \mathbf{b}^{*}$ hexagonal axes, as explained later in the text. In (a) and (b) they propagate either along the aperiodic channel direction with a longitudinal (a) or transverse polarization (b) or in the commensurate basal plane with longitudinal (c) or out-of-plane transverse polarization (d). In (c), acoustic (blue peaks) and optic (yellow peaks) modes are visible whereas in the other panels only acoustic modes are observed. Notice the log scales in (a), (b), and (d).

plane along the $\left(\mathbf{a}^{*}+\mathbf{b}^{*}\right)$ direction $(\mathbf{q} \| \Gamma K)$, a longitudinal acoustic (LA) in Figs. 3(a) and 3(c), a transverse acoustic (TA) in Figs. 3(b) and 3(d), whereas an optic mode about $10 \mathrm{meV}$ in Fig. 3(c) shows an intensity increase when $q$ goes towards the zone boundary, unlike the acoustic modes becoming less visible at large $q$ values.

\section{DYNAMICAL MODEL}

Aperiodic composites are intergrowth intermodulated crystals and treating separately the host and guest subsystems is a crude assumption. However, since the intermodulation Bragg peaks are rather weak at room temperature, two different sets of phonons can be considered in a first approximation with the present energy resolution. This is, however, not possible at the lowest energies, where excitations may reveal very specific features such as gaps opening and/or acoustic phonon overdamping. As the alkane sublattice can be simply described by a single molecule per alkane cell with a sixfold orientational disorder around the channel axis, the frequency dispersion along this direction reduces to a single branch characterized by the intermolecular force constant that has been indirectly determined [35]. This dispersion branch appears later on in Fig. 7. Other alkane modes such as librations, translations, and more localized modes seem to occur only in the diffuse scattering $D$ bands. The remaining modes seem to involve only the urea channel dynamics.

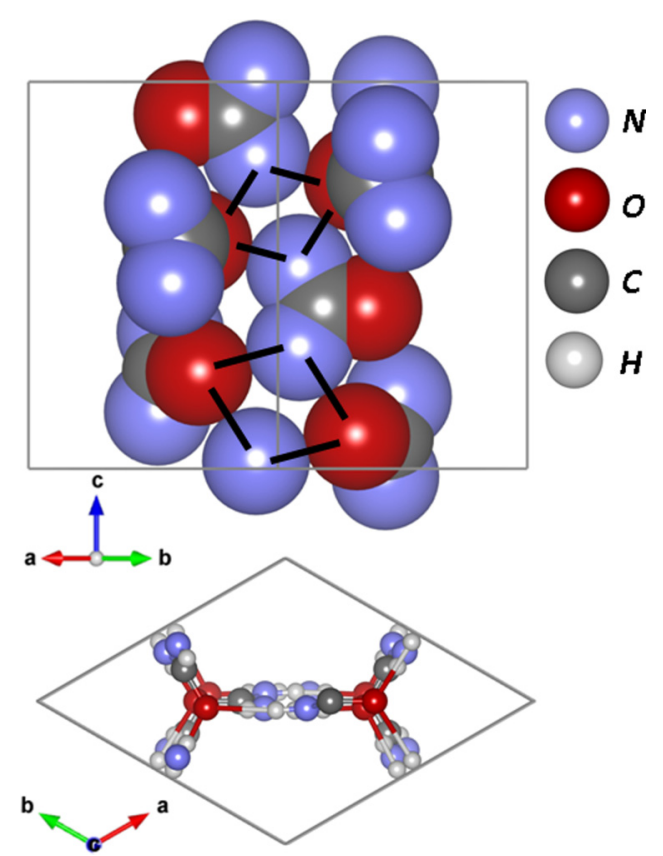

FIG. 4. Ball-and-stick diagrams of urea channels wall from the side $(\mathbf{a}+\mathbf{b})$ (top panel) and along $\mathbf{c}$ (lower panel). In the top panel, atoms are inflated to their maximum size in order to improve a $3 \mathrm{D}$ visualization of the wall that is further enhanced by black lines showing the orientation of the hydrogen bonds between oxygen and nitrogen atoms. The upper four nitrogen atoms pointing towards the viewer and the lower four pointing backwards belong to adjacent walls. The $\mathbf{c}$ view shows hydrogen atoms (in white) with smaller sized balls, allowing one to distinguish the different locations of oxygen atoms at each channel corner.

In order to describe the different experimental frequencies and understand their dispersion, it is necessary to use the result of lattice dynamics calculations. Standard dynamical calculations for urea would involve six molecules perturbed by a strong guest disorder in an aperiodic environment. A complete description, even restricted to external modes, would be an intractable task. For a partial description, simplifications are necessary. At a simpler level, we will first ignore the aperiodicity, allowing the use of Brillouin zones for this sublattice and, second, try to decouple different directions. We have calculated the dispersion curves using two basic models mimicking the structure in the basal plane and along the channel axis.

The structure of these compounds is characterized by the honeycomb shape of the urea sublattice inside which $n$ alkanes are densely packed. Honeycomb walls are made of a network of urea molecules tethered by hydrogen bonds, as shown in the top panel of Fig. 4. The hexagonal structure $P 6_{1} 22\left(D_{6}^{2}\right)$ contains in its primitive cell three of the six walls of a urea channel built by its six-urea molecules. On each channel corner, three molecules, slightly canted from the channel axis, stack their oxygen atom nearly above each other along this axis in order to realize hydrogen bonds with the nitrogen atoms of urea molecules belonging to the neighboring corner. A three-helices picture is necessary to respect the oxygen positions since two of them lie in the wall, 
two others are slightly above it, whereas the remaining two are slightly below, as shown in the $\mathbf{c}^{*}$ view of Fig. 4.

Urea molecules occupy $C_{2}$ sites with a lower symmetry than the one commonly admitted for the molecule $\left(C_{2 v}\right)$. This produces mixing of the molecular rotations $R_{j}$ and translations $T_{i}$ in the dynamic representation. Since we are only interested in lattice modes resulting from these motions along the three spatial directions, we may write the decomposition of the urea external mode representation at the zone center on the $D_{6}$ irreducible representations as

$$
\Gamma_{\text {ext }}=2 A_{1}+4 A_{2}+2 B_{1}+4 B_{2}+6 E_{1}+6 E_{2} .
$$

This formula includes the urea sublattice acoustic modes of symmetry $A_{2}$ and $E_{1}$ since they are related only to a sublattice and not to the whole crystal. This decomposition shows that each irreducible representation involves pairs of molecular translations and librations: one for $A_{1}$ and $B_{1}$ both polarized along the channel axis, two for $A_{2}$ and four for $B_{2}$ polarized in the basal plane, and three for $E_{1}$ and $E_{2}$ involving all directions. It should be pointed out that in the lowest-symmetry points of the Brillouin zone, 36 different frequencies are expected in the external modes ranging below $30 \mathrm{meV}\left(240 \mathrm{~cm}^{-1}\right)$, whereas the apparatus linewidth cannot resolve more than 24 lines within this window without any broadening. Therefore, some observed peaks, probably the broadest, are unresolved multiplets, even along symmetry directions. However, the inelastic structure factor decreases the number of visible modes by sorting them out with respect to their polarization.

The study of all these modes is outside the scope of this paper due to obvious experimental limitations, and to the fact that we are mainly interested in the lower branches, that are more likely to present features directly related to aperiodicity. Another major simplification occurs when replacing urea molecules by point masses restricting the analysis to translational modes. By doing so, we divide by two the number of computed modes. In the basal plane projection, this structure looks somewhat similar to the familiar honeycomb lattice of graphene, even if the symmetries are a bit different. In particular, the absence of a mirror plane perpendicular to the sixfold axis in this aperiodic structure does not, contrary to graphene, allow the dynamics decoupling between the inand out-of-plane motions. Numerous theoretical studies on graphene dynamics have been reported in the literature, as, for example, in Ref. [36] and references cited therein. For our calculation of the dispersion branches along symmetry directions, we chose the convenient formulation of Adamnyan and Zavalniuk [37] with two force constants $\left(J_{1}, J_{2}\right)$. It involves the first- and second-nearest-neighbor's central forces in order to stabilize the lattice against a collapsing shear. Within this model, the second-neighbor interaction in graphene stabilizes the lattice, while in urea inclusion compounds this effect is likely obtained by an alkane guest nearest neighbor, via van der Waals forces and/or steric hindrance. Another lattice dynamics model of urea channels has been previously reported with some success [38], using two force constants along the channel direction, but without any second-order neighbor in the basal plane, precluding the study of transverse acoustic phonons polarized in this plane. Here, we describe the out-ofplane transverse modes $\mathrm{TA}_{z}$ and $\mathrm{TO}_{z}$, by the simplest formula
TABLE I. Fit parameters for the dispersion of the different branches obtained either by giving the maximum frequency of the optic branch or by their initial slope at infinite wavelengths $(q \sim 0)$. The first index labeling transverse modes defines the propagation direction whereas the second one is for polarization. For $L_{K M}$ and $T_{x y}$ modes in the hexagonal plane, the ratio of the second- to the first-neighbors' force constants $J_{2} / J_{1}$ is equal to 0.12 .

\begin{tabular}{lccccc}
\hline \hline Disp. branch & $L_{K M}$ & $T_{x z}$ & $T_{x y}$ & $L_{z}$ & $T_{z x}$ \\
\hline Max. freq. (meV) & 13.7 & 8.57 & 13.7 & 21.0 & 6.9 \\
Sound velocity $\left(\mathrm{km} \mathrm{s}^{-1}\right)$ & 3.64 & 2.18 & 1.36 & 5.84 & 1.87 \\
\hline \hline
\end{tabular}

compatible with hexagonal symmetry,

$$
\Omega_{Z \pm}=\Omega_{0}\left(1 \pm \sqrt{\frac{3+F_{0}(\mathbf{q})}{3}}\right)^{1 / 2}
$$

where $\Omega_{0}$ is the maximum frequency, with $F_{0}(\mathbf{q})=$ $2\left[\cos \left(q_{1} a-q_{2} a\right)+\cos \left(q_{1} a\right)+\cos \left(q_{2} a\right)\right]$, with q being the phonon wave vector of components $\left(q_{1}, q_{2}\right)$ parallel to the reciprocal hexagonal basis vectors $\left(\mathbf{a}^{*}, \mathbf{b}^{*}\right)$. Consequently, our description will be limited to in-phase (LA, TA) and outof-phase motions (LO, TO) in three directions of the two infinitely rigid stacks of the primitive cell, requiring only three adjustable parameters to describe six dispersion branches (LA, LO, TA, TO, $\mathrm{TA}_{z}, \mathrm{TO}_{z}$ ).

Along the perpendicular direction, the main structural feature is the presence of three urea helices described above, resulting in a triple folding of the dispersion branches eventually with no gap. Henceforth, we have described them with a linear chain model with three identical masses per cell, each built by two urea molecules moving in phase in the primitive cell. In contrast, the motions considered in the basal plane involve relative displacements inside the helices, so we cannot expect any description along the general directions of propagation in the scattering plane. However, these simple models describe the shape of the phonon branches even at $30^{\circ}$ away from the principal directions. Noting that transverse modes are degenerate due to the sixfold symmetry, we use models with three adjustable parameters in the $\left(\mathbf{a}^{*}, \mathbf{b}^{*}\right)$ plane and two along the $\mathbf{z}$ directions for 17 dispersion segments between the high-symmetry points of the first Brillouin zone. These parameters can be expressed either by the maximum frequency of the highest optic branch or by the initial slope of the acoustic one, when knowing the mass multiplicity along any direction (see Table I). In the commensurate plane these parameters, computed from the first- and second-neighbor's force constants in graphene, are found to have a ratio of about $1: 8$ that is in accordance with a rather strong hydrogen bond between urea molecules and a van der Waals interaction between urea and nearest-neighboring alkane.

Since spectra have been recorded around many reciprocal locations, the variation of inelastic intensities for the same mode provides information on its polarization e due to its inelastic scattering structure factor and allows some discrimination. Theoretically, any mode perpendicular to the scattering plane should not appear in the spectra if recorded with a perfect collimation. This is the case for the transverse modes 


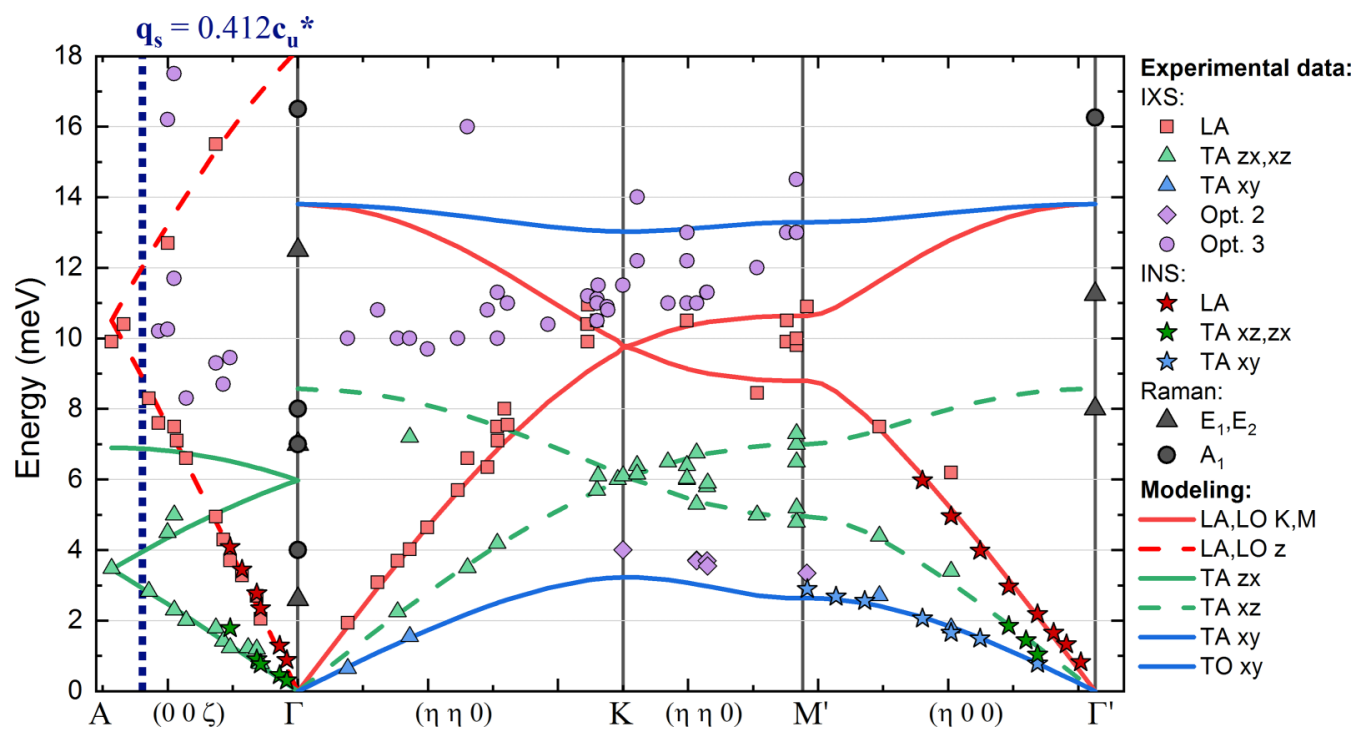

FIG. 5. Dispersion of the lower phonon branches along the channel direction $(\Gamma A)$ at the left and along the principal directions of the basal plane $\left(\Gamma K M^{\prime}\right)$ in the middle and $\left(M^{\prime} \Gamma^{\prime}\right)$ at the right. The dotted vertical line close to $A$ indicates the wave vector of the intermodulation $\mathbf{q}_{\mathrm{s}}=0.412 \mathbf{c}_{\mathrm{u}}^{*}$. The lengths of the segments $A \Gamma, \Gamma K, K M^{\prime}$, and $M^{\prime} \Gamma^{\prime}$ are proportional to their absolute values, so that sound velocities along these directions are visually comparable. Notice that the tick origin lies at $\Gamma$. Solid data points come from IXS and asterisks from INS [20]. Colors of the points and lines are listed in the legend. Black circles at the zone center $\Gamma$ are Raman frequencies of the urea sublattice [39]. The points on the left axis are for the $A_{1}(\bullet)$ and $E_{1}(\boldsymbol{\Delta})$ symmetries polarized completely or partially along $z$, whereas at the far right, for frequencies assigned with the $A_{1}(\bullet)$ and $E_{2}(\boldsymbol{\Delta})$ symmetries polarized in the basal plane $\left(1 \mathrm{meV}=8.06 \mathrm{~cm}^{-1}=242 \mathrm{GHz}\right)$.

polarized in the basal plane since most of the time we used the $\left(\mathbf{a}^{*}+\mathbf{b}^{*}, \mathbf{c}^{*}\right)$ scattering plane.

\section{PHONON DISPERSION}

Although many spectra were recorded in the whole cross section of the urea first Brillouin zone, we will mainly focus on the presentation of dispersion branches of phonons propagating along the symmetry directions $\Gamma A$, where $\mathbf{q}=q\left(\mathbf{c}_{\mathbf{u}}^{*}\right)$, $\Gamma K$ with $q_{1}=q_{2}=q$ and $\Gamma M$, where $q_{1}$ or $q_{2}$ equals zero. Figure 5 gathers all the frequencies of the DHOs found in the fit of inelastic spectra for propagation in the main directions of the basal plane on the right-hand side, and along the channels on the left one. Many data points may be interpreted by the dispersion models described above. However, other points obviously do not belong to these branches but to other vibrations, possibly librations. It should be noted that phonons polarized in the commensurate $(\mathbf{a}, \mathbf{b})$ plane (drawn by solid lines) involve both sublattices, in contrast to those polarized along the channel direction (drawn by dashed lines), which, due to aperiodicity, may involve mainly one sublattice.

Along $\Gamma K$, the longitudinal acoustic mode and the transverse one polarized along $\mathbf{c}^{*}$ have been studied close to the more intense (2200) Bragg reflection. This direction corresponds, in real space, to the normal to the hexagon sides, along which a unique periodicity appears. Consequently, no gap in the related dispersion branches is either expected or observed within the resolution $(\sim 1 \mathrm{meV})$ and the scarcity of points at the zone edge. About half of these points come from (2200) reflection [Figs. 3(c) and $3(\mathrm{~d})$ ], while the others result from different scattering geometries in the same scattering plane. Two points (blue triangles, $\mathrm{TA}_{x y}$ ) seem related to the transverse acoustic mode polarized in the basal plane that should not contribute to the scattered intensity; however, they are not well resolved in the spectra and only appear from the best fits with a quite weak intensity.

Beyond $K$, propagation occurs on the $K M^{\prime}$ zone edge with no obvious changes with respect to the $\Gamma K$ branch, but new data points appear close to the lowest dispersion branch. These points have been recorded around an $M$ point at the (1.5 1.530$)$ location, close to the critical wave vector of the lowest-temperature phase where previous observations of low-frequency dynamics could be observed. Although the frequency of these modes is very close $(1 \mathrm{meV})$ to the lowest acoustic branch, such an assignment is impossible for several reasons. First, it would correspond to an increase of the transverse sound velocity by more than $20 \%$ that is inconsistent with neutron and Brillouin scattering measurements, or with the sixfold symmetry. Second, and more conclusive, they are very intense, weakly damped, and unique in their frequency range, as shown in Fig. 6(a), suggesting that their polarization belongs rather to the $\left(\mathbf{a}^{*}+\mathbf{b}^{*}, \mathbf{c}^{*}\right)$ scattering plane than to the $\left(\mathbf{a}^{*}-\mathbf{b}^{*}\right)$ direction, mandatory for this transverse branch. This mode may be followed for $q_{z} \neq 0$ as shown in Fig. 6(b), where its dispersion is shown along $K H, S T$, and $M L ; K M$ presented in Fig. 5. We observe a weaker dispersion along the c $^{*}$ in the neighborhood of $M$ than in the vicinity of $K$ that is in agreement with a structural phase instability at $M \pm 0.09 \mathbf{c}_{\mathbf{u}}^{*}$ at lower temperatures, practically without any vibrational energy cost.

Along the $\Gamma M$ direction, only two experiments were performed by tilting the sample, resulting in a poorer resolution at $\mathbf{q}=\Gamma M / 2$ and $\mathbf{q}=(3 / 4) \Gamma M$. In real space, this corresponds to a propagation along the direction where short and long periods alternate, leading to a frequency gap between the 

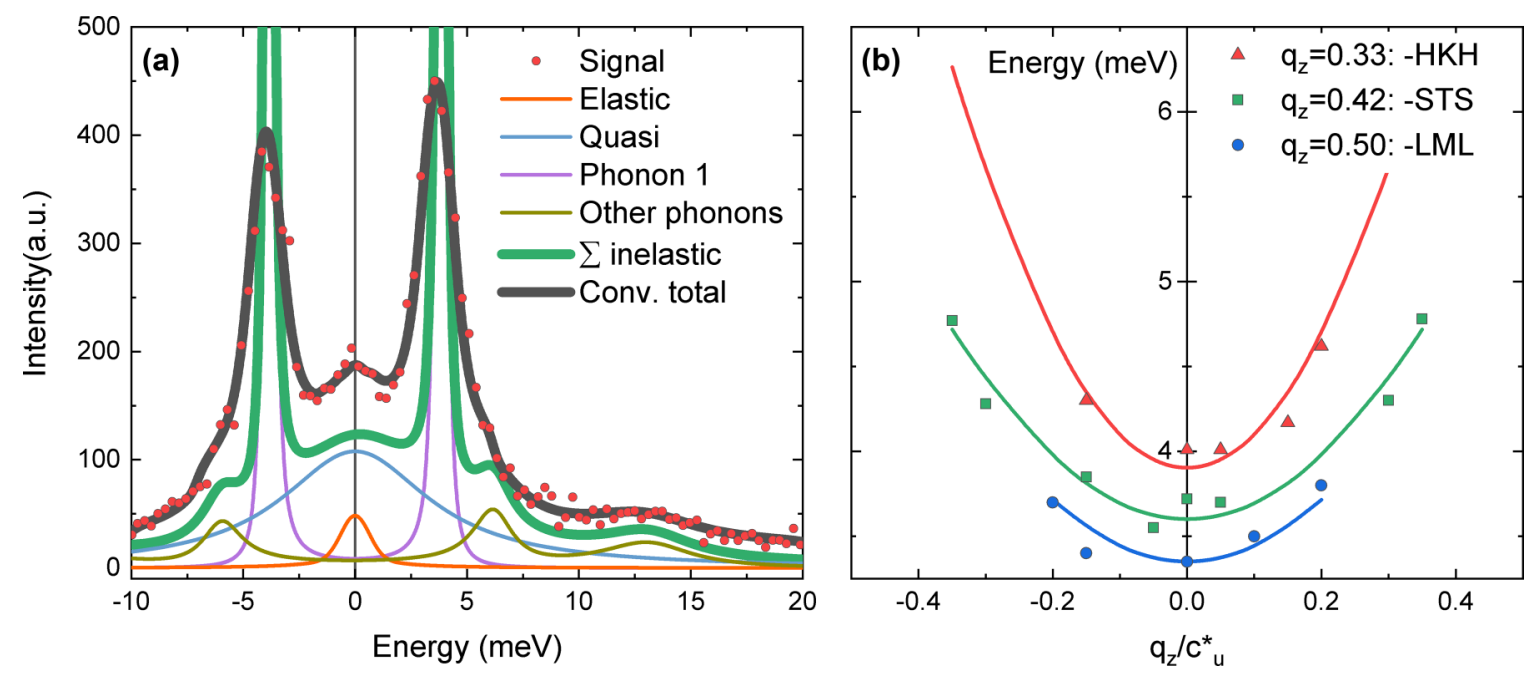

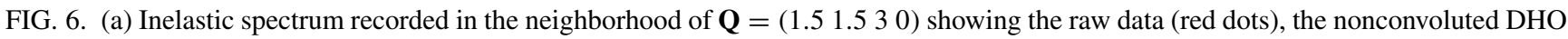
profiles in green lines, and a quasielastic profile in blue. The sum of these inelastic contributions weighted by the Bose factor is in gray whereas the elastic contribution in orange is added to the convoluted spectrum in bold black. (b) Zone edge dispersion of the related phonon branches along $\mathbf{c}^{*}$. The lowest branch in blue is along $-L M L$ at $q_{z}=-0.5,0,+0.5$, respectively, whereas the highest branch in red corresponds to $-H K H$ and the intermediate branch in green to $-S^{\prime} T^{\prime} S^{\prime}$ at a location halfway between $M$ and $K$. The frequency (3.85 meV) from the spectrum on the left is located on this curve at $\mathbf{q}_{\mathbf{z}} / \mathbf{c}^{*}=-0.14$.

optic and acoustic branches. This geometry with $\mathbf{Q}=(1.5-$ $x) \mathbf{a}^{*}+(1.5+x) \mathbf{b}^{*}+3 \mathbf{c}_{\mathbf{h}}^{*}$ generates a phonon wave vector with a component parallel to the $\left(\mathbf{a}^{*}-\mathbf{b}^{*}\right)$ direction equal to $x \mathbf{a}^{*}$ that activates the lowest transverse mode polarized along $\left(\mathbf{a}^{*}-\mathbf{b}^{*}\right)$, whereas the longitudinal acoustic mode almost vanishes. This propagation direction was previously investigated by inelastic neutron scattering (INS) for the acoustic branches [20]. The related data points $(\star)$ reported in Fig. 5 are in good agreement with the present results. Here, Raman frequencies are close to the gap edges where flat dispersion branches present a high density of states and are not very far from the LO maximum calculated at $13.8 \mathrm{meV}$ and found at $16 \mathrm{meV}$ by Raman scattering [39]. At higher frequencies, Raman lines about $20 \mathrm{meV}$ could also be assigned to librational modes as in pure quadratic urea [40].

Along $\Gamma A$, longitudinal and transverse acoustic modes have been investigated around the strongest reflections along $\mathbf{c}_{\mathbf{u}}^{*}\left(\begin{array}{llll}0 & 0 & 6 & 0\end{array}\right)$ and $\left(\begin{array}{llll}0 & 0 & 0 & 12\end{array}\right)$, the latter visible in Figs. 3(a) and 3(b). Along this direction, dispersion branches may be assumed to be triply folded when ignoring perturbations by libration branches as explained above. At this resolution, no additional acousticlike branch appears, as could be expected in case of an underdamped sliding mode. As for several spectra, quasielastic components with a width in the $2-\mathrm{meV}$ range have been included in the fits of spectra recorded around (0060). Such contributions with a similar width are not so astonishing, when one considers the amount of disorder inside this compound. Moreover, it has been previously shown that the $D$ bands have a partial dynamical origin such as the pretransitional diffuse scattering [41].

Obviously, other modes appear in the diagram (circles) in the 10-meV range that are not described by the few computed branches. However, it is interesting to notice that our simple model could explain the origin of the three lowest Raman frequencies on the left ordinate axis. Their frequencies are very close to the values of the three transverse branches at the intersection occurring at $\mathbf{q}_{\mathbf{s}} \approx 0.412 \mathbf{c}_{\mathbf{u}}^{*}$, which activates Raman scattering as a zone center. It also appears that the two frequencies above could be activated on the longitudinal optic mode for the same reason.

\section{APERIODIC FEATURES}

At first sight, one might think that these dispersion curves are similar to those of a regular periodic crystal, but that is not the case if we look more thoroughly at the phonon branches polarized along $\mathbf{c}^{*}$ (dashed lines in Fig. 5). First, in the unlabeled composite, the dispersion curve of the longitudinal mode along $\mathbf{c}^{*}$ has a slope of $5.84 \mathrm{~km} \mathrm{~s}^{-1}$. This value is much larger than the sound velocity of the $n$-nonadecane/urea measured by Brillouin scattering of $4.8 \mathrm{~km} \mathrm{~s}^{-1}$ [42] or in other $n$-alkane/urea compounds such as $C_{12}$ [42] or $C_{20}$ and $C_{22}$ in Ref. [43]. Obviously, with the possibility to sort out the sublattices along this direction, it seems reasonable to assign this larger value to the stiffer honeycomb urea sublattice. Due to its instability with respect to the noninclusion of guests, we may only compare it with sound velocities of the tetragonal urea phase, where ultrasonics [44], Brillouin scattering [45], and INS [40] investigations reasonably agree on a $C_{33}$ value around 51(2) GPa. As shown in Table II, such a value corresponds to sound velocities of about $6.2 \mathrm{~km} \mathrm{~s}^{-1}$ in unlabeled urea with a mass density of $1.334 \mathrm{~kg} \mathrm{~m}^{-3}$, or $5.9 \mathrm{~km} \mathrm{~s}^{-1}$ in the deuterated crystal with a heavier density of $1.423 \mathrm{~kg} \mathrm{~m}^{-3}$.

Despite a different packing in tetragonal and honeycomb lattices, the orientation of hydrogen bonds is comparable, leading to a similar anisotropy of the elastic properties that makes some sense in this comparison. For completeness, we also report data related to the $n$-nonadecane/urea composite, both for the urea sublattice by INS and the whole composite by Brillouin scattering. We notice that the velocity in the deuterated urea sublattice measured by INS [20] at $160 \mathrm{~K}$ is equal to the one of unlabeled tetragonal urea and larger than 
TABLE II. Mass densities and longitudinal velocities in pure tetragonal urea, urea hexagonal sublattice, and in an $n$ nonadecane/urea composite for deuterated and unlabeled species. The $300-\mathrm{K}$ value of the deuterated hexagonal sublattice is computed from an anharmonic variation, as described in the text.

\begin{tabular}{lcc}
\hline \hline Urea & $\begin{array}{c}\text { Mass density } \\
\left(\mathrm{kg} \mathrm{L}^{-1}\right)\end{array}$ & $\begin{array}{c}\text { Highest longitudinal } \\
\text { velocity }\left(\mathrm{km} \mathrm{s}^{-1}\right)\end{array}$ \\
\hline Tetragonal $H$ & 1.334 & $6.2[44,45]$ \\
Tetragonal $D$ & 1.423 & $5.9[40]$ \\
Hexagonal sub $H$ & 0.933 & 5.8 \\
Hexagonal sub $D$ 150-300 K & 0.995 & $6.2[20]-(5.6)$ \\
C-19 urea $H$ & 1.224 & $4.8[42]$ \\
C-19 urea $D$ & 1.328 & \\
\hline \hline
\end{tabular}

the unlabeled urea sublattice velocity in the present study at $300 \mathrm{~K}$. However, we also performed some IXS experiments at the same temperature and observed a $9 \%$ increase of the velocity of this mode with respect to the $300-\mathrm{K}$ value. If we apply this anharmonic correction to the deuterated crystal, we obtain a velocity of $5.6 \mathrm{~km} \mathrm{~s}^{-1}$ at room temperature that is in very good agreement with the present value, taking into account the change of mass.

It is also noteworthy that this anharmonic relative variation of the urea sublattice longitudinal velocity is exactly the same as the one measured by Brillouin scattering on the composite longitudinal acoustic mode, while it is more than twice smaller in tetragonal urea [45]. This equality strongly suggests a common origin lying in the anharmonicity of the urea hydrogen bonds, which can be considered as the main elastic interactions in the whole composite structure. Then, in a first approximation, we could expect to explain these different sound velocities in the composite only by different mass densities in the equation $\sqrt{\left(\rho_{1}+\rho_{2}\right)} V_{-}=\sqrt{\rho_{1}} V_{1}$, where $\rho$ is a mass density, $V$ a velocity, and subscripts 1 or 2 refer to urea and alkane, respectively, while $V_{-}$is the sound velocity of the composite. Consequently, the ratio $V_{-} / V_{1}=0.828$ should be equal to the square root of the related densities equal to 0.873 . For such a simple approximation, the agreement is reasonable; however, it could be improved by taking into account the intermodulation of sublattices. For example, a fraction of the urea sublattice modulated with the alkane period results in a urea effective mass density lower than the uncoupled one. If sufficiently smooth, this intermodulation may also produce the occurrence of a dynamic phase mode (or sliding mode), which in the case of a rather unlikely underdamped regime would generate a symmetric doublet in inelastic spectra at the lower wave vectors. In the frame of a harmonic double-chain model, Radulescu and Janssen express the sound and sliding mode velocities by the following equation [46],

$$
V_{ \pm}^{2} \approx \frac{1}{2}\left(V_{1}^{2}+V_{2}^{2}\right)\left[1 \pm \frac{\left(V_{1}^{2}-V_{2}^{2}\right)\left(\rho_{1}-\rho_{2}\right)}{\left(V_{1}^{2}+V_{2}^{2}\right)\left(\rho_{1}+\rho_{2}\right)}\right],
$$

where $V_{2}$ is the sound velocity of the alkane sublattice.

This formula agrees with the anharmonic description of Finger and Rice for the sound velocity whereas their sliding mode is relaxational [19]. We have plotted the dispersion of these related quantities in Fig. 7 after having computed the

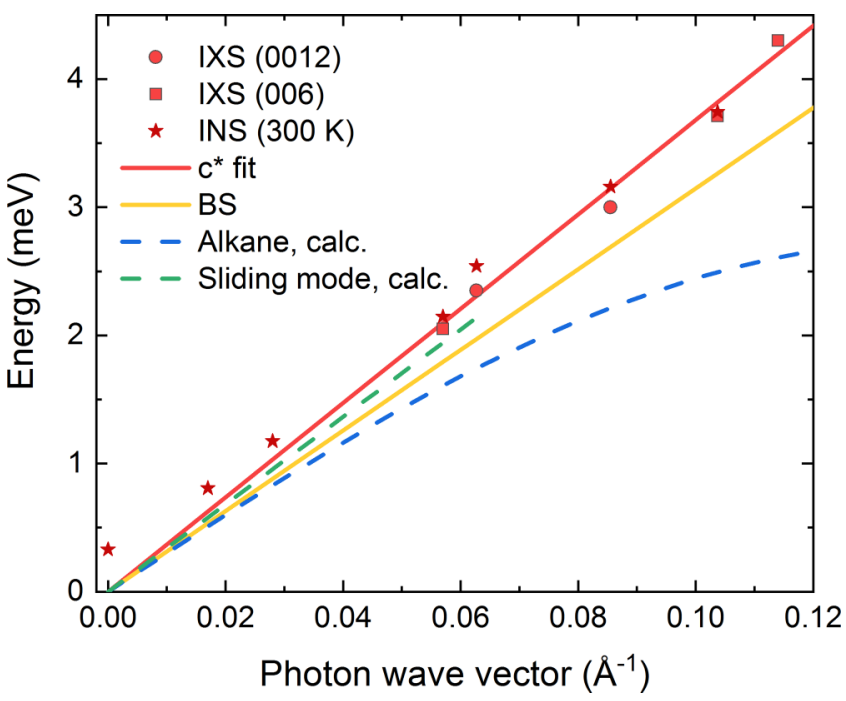

FIG. 7. Dispersion of longitudinal acoustic modes along the $\mathbf{c}^{*}$ axis. Measured IXS dispersion profile $\left(V_{1}\right)$ and the initial slope from Brillouin scattering (BS: $V_{-}$) data are in solid red and orange lines, respectively, whereas the initial slope of the hypothetical sliding mode $\left(V_{+}\right)$and the calculated velocity of the alkane sublattice $\left(V_{2}\right)$ are drawn in green and blue dashes, respectively. Solid symbols and asterisks refer to IXS and INS data corrected for anharmonic variation.

alkane sublattice longitudinal velocity $V_{2}=4 \mathrm{kms}^{-1}$ from the force constant obtained by a specific compressibility experiment [35]. It gives 4.7 and $5.2 \mathrm{~km} \mathrm{~s}^{-1}$ for $V_{-}$and $V_{+}$that is in close agreement, for the lowest value, with the composite acoustic velocity. A perfect agreement would be obtained by an increase of the alkane velocity up to $4.1 \mathrm{~km} \mathrm{~s}^{-1}$ yielding to a sliding mode velocity of $5.3 \mathrm{~km} \mathrm{~s}^{-1}$. Regardless, this indicates that in inelastic scattering experiments both modes would lie at close frequencies in the absence of defects and singularities pinning the modulation.

However, this condition is not obvious since under pressure, selective compressibility of sublattices occurs only above a threshold pressure about $1 \mathrm{kbar}$ corresponding to a rather low-energy barrier in the $0.1-\mathrm{meV} /$ guest range, more likely related to scattered defects along the channels than to intrinsic singularities of the intermodulation, repeating all along the channels. As previously noted, the alkane sublattice presents along the channel direction a simple dispersion for the longitudinal mode with a slope at the origin defined by $V_{2}$, and reaching a maximum value of $2.7 \mathrm{meV}$ around $0.13 \AA^{-1}$ at its zone boundary as displayed in Fig. 7. This value is very close to the highest frequency previously detected in the decomposition of the $D$ planes' diffuse scattering [41]. Since this diffuse scattering is expected to be due to librations or translations of the center of mass of the molecules, the agreement between these values suggests the highest component could be related to longitudinal translations. This maximum value, associated with a high density of states, is also very commonly found in the fits of quasielastic components of our IXS spectra.

Due to symmetric strain and stress tensors, the transverse acoustic waves propagating along directions where a single elastic constant is involved, have a velocity equal to the one 
of the mode propagating along the polarization direction of the former and polarized along its propagation direction. Such directions occur in the case of orthogonal symmetry axes and, in particular, in any plane containing the sixfold axis in hexagonal crystals. This velocity, equal to $\sqrt{\frac{C_{44}}{\rho}}$ in the Voigt notation, is expected for a propagation along this axis as well as for any perpendicular direction to this axis [47]. This is related to the equality of the shearing force constants occurring along these directions in usual periodic crystals.

As already pointed out [18], aperiodic host-guest structures could break this symmetry. The modes, possibly anomalous with respect to regular hexagonal periodic crystals, are the transverse acoustic polarized and propagating in planes containing the $\mathbf{c}^{*}$ direction. The anomaly rests upon the structural difference between, on the one hand, a TA mode polarized perpendicular to $\mathbf{c}^{*}$ with equal displacements of the molecules of both sublattices as in a regular crystal and, on the other hand, a propagation of the TA mode in the perpendicular plane with a polarization along $\mathbf{c}^{*}$. In that case, the absence of restoring forces between sublattices allows independent translations of the sublattices. This yields to different velocities which break the rotational symmetry since the molecular packing is different along $\mathbf{c}^{*}$ and any direction in the basal plane, as sketched in Fig. 1. Consequently, the transverse velocity will be larger for single urea displacements along the incommensurate direction than the one with displacements of the whole crystal in the $\left(\mathbf{a}^{*}, \mathbf{b}^{*}\right)$ plane. Here, again, the ratio of the velocities of these modes should be equal to the reciprocal ratio of the square root of the involved densities as for the longitudinal mode. Figure 8 gathers IXS results and the previous BS [42] and INS results [20]. The neutron values have been corrected from a checked 9\% anharmonic variation. Although these velocities are rather close to the uncertainty limit, they are supported by two very similar observations previously realized by different inelastic

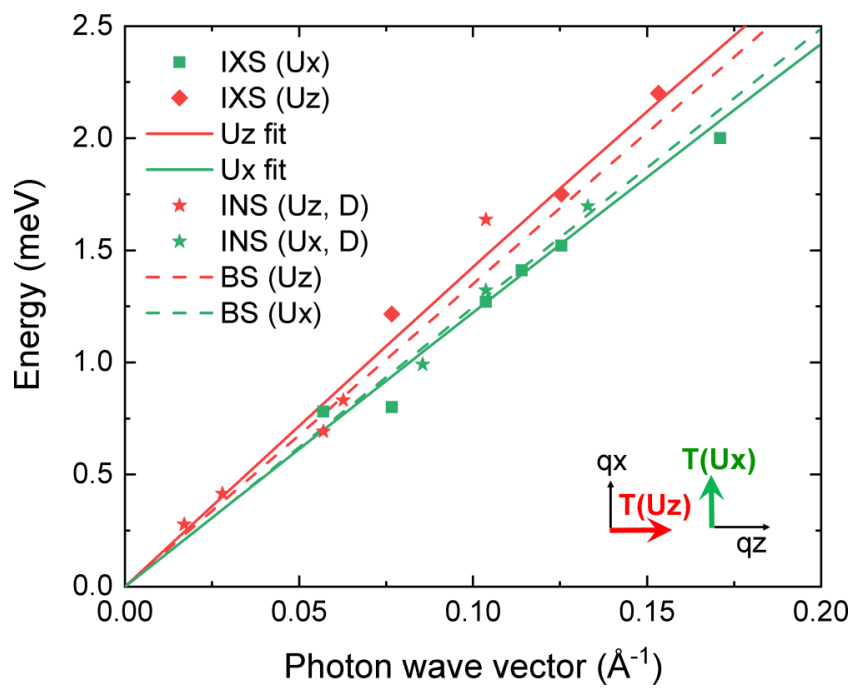

FIG. 8. Dispersion of transverse acoustic modes in the $\left(\mathbf{a}^{*}+\right.$ $\left.\mathbf{b}^{*}, \mathbf{c}^{*}\right)$ plane, polarized along $\mathbf{c}^{*}$ labeled $T\left(U_{z}\right)$ in red, and polarized along $\left(\mathbf{a}^{*}+\mathbf{b}^{*}\right)$ labeled $T\left(U_{x}\right)$ in green. Solid lines are related to calculated dispersion from the lattice dynamics model, dashed lines to Brillouin scattering measurements, solid symbols to IXS, and asterisks to INS corrected of anharmonic variation. $D$ in the legend is related to a deuterated crystal. techniques. In each case, the difference between these two modes was at the limit of the error range so we adopted the same velocity, $2.0 \pm 0.1 \mathrm{~km} \mathrm{~s}^{-1}$, for these modes. However, it should be noted that this rather small difference always presents the same sign with a larger velocity for a polarization along the aperiodic direction. Such a reproducibility for three different techniques with different samples has a poor probability of $12 \%$ in the case of a random origin. Consequently, we may say with a confidence close to $90 \%$ that this difference has a physical origin. Among the possible origins, piezoelectricity may stiffen crystals along some directions. It can easily be ruled out since in 622 symmetries, only two opposite piezoelectric constants $e_{123}=-e_{213}$ are nonzero but they are inactive along principal axes [48]. With velocities of $2.18 \mathrm{~km} \mathrm{~s}^{-1}$ for $T\left(U_{z}\right)$ and $1.87 \mathrm{~km} \mathrm{~s}^{-1}$ for $T\left(U_{x}\right)$ as quoted in Table I, their ratio of 0.858 is very close to the square root of masses (0.873) and strongly suggests an aperiodic physical origin. Nevertheless, the small difference does not preclude any intermodulation affecting the effective masses, although to a lesser extent. Moreover, experimental uncertainties are not negligible at this scale.

\section{CONCLUSION}

In summary, we have reported an extended study of phonons in an aperiodic composite of $n$-nonadecane/urea. The use of inelastic x-ray scattering has allowed for a rather complete description of the rigid host sublattice. A combined analysis of these results with coherent cold neutrons and Brillouin scattering data provide insight into the complicated topics of collective vibrations in crystals without an overall Brillouin zone. A large number of dynamical modes are now observed and identified along several directions. Despite the disorder of the guest, it was possible to sort the dynamic features of each sublattice.

In the vicinity of common Bragg peaks, we have detected phonons of the whole composite structure which, for the acoustic mode, have a dispersion in agreement with previous BS and INS measurements. Those polarized in the hexagonal commensurate plane do not exhibit anomalies whereas the transverse acoustic one polarized along the channel presents a slightly larger velocity than the transverse one propagating along the channel direction and polarized in the hexagonal plane. This small deviation is at the error limit but, since the same deviation has been also observed by previous Brillouin scattering and inelastic neutron scattering experiments, it is reasonable to assign, with a large probability, this difference to an elasticity symmetry breaking predicted in aperiodic composite structures. This difference is generated by the difference of mass densities of the involved urea and composite lattices. This is one of the rare observations of this elastic symmetry breaking in aperiodic structures such as in mercury chains [14] or spin-ladder systems [10] with some differences due to the nature of the sublattices.

\section{ACKNOWLEDGMENTS}

The authors acknowledge P. Breczewski for the growth of very large good-quality crystals, P. Bourges and $\mathrm{M}$. de Boissieu for stimulating and enlightening scientific 
discussions, and D. de Sanctis for allowing measurements on the ID29 ESRF beamline. A part of this work also received the support from the European Union's Horizon 2020 re- search and innovation program under the Marie SkłodowskaCurie Grant Agreement No. 701647 and SNSF Grant No. 200021L_169753.
[1] T. Janssen, G. Chapuis, and M. de Boissieu, Aperiodic Crystals: From Modulated Phases to Quasicrystals (Oxford University Press, Oxford, UK, 2007), pp. 1-480.

[2] T. Janssen, A. Janner, A. Looijenga-Vos, and P. M. de Wolff, in International Tables for Crystallography (International Union of Crystallography, Chester, UK, 2006), pp. 907-955.

[3] R. Blinc and A. P. Levanyuk, Incommensurate Phases in Dielectrics, Part 1, Modern Problems in Condensed Matter Sciences Vol. 14 (North-Holland, Amsterdam, 1986).

[4] J. D. Axe, M. Iizumi, and G. Shirane, Phys. Rev. B 22, 3408 (1980).

[5] R. Blinc and A. P. Levanyuk, Incommensurate Phases in Dielectrics, Part 2, Modern Problems in Condensed Matter Sciences Vol. 14 (North-Holland, Amsterdam, 1986).

[6] H. Z. Cummins, Phys. Rep. 185, 211 (1998).

[7] J. Ollivier, J. Etrillard, B. Toudic, C. Ecolivet, P. Bourges, and A. P. Levanyuk, Phys. Rev. Lett. 81, 3667 (1998).

[8] J.C. Toledano and P. Toledano, The Landau Theory of Phase Transitions, World Scientific Lecture Notes in Physics Vol. 3 (World Scientific, Singapore, 1989).

[9] O. Radulescu, T. Janssen, and J. Etrillard, Eur. Phys. J. B 29, 385 (2002).

[10] X. Chen, D. Bansal, S. Sullivan, D. L. Abernathy, A. A. Aczel, J. Zhou, O. Delaire, and L. Shi, Phys. Rev. B 94, 134309 (2016).

[11] T. Janssen, O. Radulescu, and A. N. Rubtsov, Eur. Phys. J. B 29, 85 (2002).

[12] L. R. José, C. McCabe, and T. C. Peter, Nanotechnology 16, 186 (2005).

[13] N. Manini, M. Cesaratto, G. E. Santoro, E. Tosatti, and A. Vanossi, J. Phys.: Condens. Matter 19, 305016 (2007).

[14] I. U. Heilmann, J. D. Axe, J. M. Hastings, G. Shirane, A. J. Heeger, and A. G. MacDiarmid, Phys. Rev. B 20, 751 (1979).

[15] I. Loa, L. F. Lundegaard, M. I. McMahon, S. R. Evans, A. Bossak, and M. Krisch, Phys. Rev. Lett. 99, 035501 (2007).

[16] J. Etrillard, P. Bourges, H. F. He, B. Keimer, B. Liang, and C. T. Lin, Europhys. Lett. 55, 201 (2001).

[17] G. Theodorou and T. M. Rice, Phys. Rev. B 18, 2840 (1978).

[18] J. D. Axe and P. Bak, Phys. Rev. B 26, 4963 (1982).

[19] W. Finger, and T. M. Rice, Phys. Rev. B 28, 340 (1983).

[20] B. Toudic, R. Lefort, C. Ecolivet, L. Guérin, R. Currat, P. Bourges, and T. Breczewski, Phys. Rev. Lett. 107, 205502 (2011).

[21] C. Mariette, L. Guérin, P. Rabiller, Y.-S. Chen, A. Bosak, A. Popov, M. D. Hollingsworth, and B. Toudic, Z. Kristallogr. 230, 5 (2015).

[22] C. Mariette, M. Huard, P. Rabiller, S. M. Nichols, C. Ecolivet, T. Janssen, K. E. Alquist, M. D. Hollingsworth, and B. Toudic, J. Chem. Phys. 136, 104507 (2012).

[23] A. El Baghdadi, E. J. Dufourc, and F. Guillaume, J. Phys. Chem. 100, 1746 (1996).

[24] R. Lefort, J. Etrillard, B. Toudic, F. Guillaume, T. Breczewski, and P. Bourges, Phys. Rev. Lett. 77, 4027 (1996).
[25] T. Weber, H. Boysen, M. Honal, F. Frey, and R. B. Neder, Z. Kristallogr. 211, 238 (1996).

[26] L. Bourgeois, B. Toudic, C. Ecolivet, J. C. Ameline, P. Bourges, F. Guillaume, and T. Breczewski, Phys. Rev. Lett. 93, 026101 (2004).

[27] C. Mariette, Ph.D. thesis, Université de Rennes 1, 2011, www.theses.fr/177175540.

[28] B. Toudic, P. Rabiller, L. Bourgeois, M. Huard, C. Ecolivet, G. J. McIntyre, P. Bourges, T. Breczewski, and T. Janssen, Europhys. Lett. 93, 16003 (2011).

[29] H. Le Lann, C. Odin, B. Toudic, J. C. Ameline, J. Gallier, F. Guillaume, and T. Breczewski, Phys. Rev. B 62, 5442 (2000).

[30] J. Ollivier, C. Ecolivet, S. Beaufils, and F. Guillaume, in Aperiodic' 97, edited by J. L. Verger-Gaugry, M. de Boissieu, and R. Currat (World Scientific, Singapore, 1997), pp. 685-690.

[31] ESRF ID28 - Inelastic Scattering II, http://www.esrf.eu/Users AndScience/Experiments/DynExtrCond/ID28/.

[32] R. Forst, H. Jagodzinski, H. Boysen, and F. Frey, Acta Crystallogr., Sect. B 43, 187 (1987).

[33] B. Toudic, H. Le Lann, F. Guillaume, R. E. Lechner, J. Ollivier, and P. Bourges, Chem. Phys. 292, 191 (2003).

[34] C. Mariette, L. Guérin, P. Rabiller, C. Ecolivet, P. GarcíaOrduña, P. Bourges, A. Bosak, D. de Sanctis, M. D. Hollingsworth, T. Janssen, and B. Toudic, Phys. Rev. B 87, 104101 (2013).

[35] L. Bourgeois, C. Ecolivet, B. Toudic, P. Bourges, and T. Breczewski, Phys. Rev. Lett. 91, 025504 (2003).

[36] L. A. Falkovsky, Phys. Lett. A 372, 5189 (2008).

[37] V. Adamyan and V. Zavalniuk, J. Phys.: Condens. Matter 23, 015402 (2011).

[38] D. Schmicker, S. van Smaalen, C. Haas, and K. D. M. Harris, Phys. Rev. B 49, 11572 (1994).

[39] M. Couzi, F. Guillaume, and T. Breczewski (private communication).

[40] J. Lefebvre, M. More, P. Fouret, B. Hennion, and R. Currat, J. Phys. C 8, 2011 (1975).

[41] R. Lefort, B. Toudic, J. Etrillard, F. Guillaume, P. Bourges, R. Currat, and T. Breczewski, Eur. Phys. J. B 24, 51 (2001).

[42] J. Ollivier, Ph.D. thesis, Université de Rennes 1, 1997, www.theses.fr/1997REN10095.

[43] J. Mielcarek, B. Mroz, T. Breczewski, and A. Trzaskowska, Ferroelectrics 334, 241 (2006).

[44] G. Fischer and J. Zarembowitch, C. R. Acad. Sci. B Phys. 270, 852 (1970).

[45] A. Yoshihara and E. R. Bernstein, J. Chem. Phys. 77, 5319 (1982).

[46] O. Radulescu and T. Janssen, J. Phys. A 30, 4199 (1997).

[47] M. J. P. Musgrave, Crystal Acoustics (Holden-Day, San Francisco, CA, 1970).

[48] C. Daros and H. Antes, Wave Motion 31, 237 (2000). 